Documentation et bibliothèques

DOCUMENTATION BIBLIOTHËQUES

\title{
Analyse du dépouillement de la revue Documentation et bibliothèques par les index
}

\section{Francine Marcouiller}

Volume 30, numéro 1, janvier-mars 1984

URI : https://id.erudit.org/iderudit/1053598ar

DOI : https://doi.org/10.7202/1053598ar

Aller au sommaire du numéro

\section{Éditeur(s)}

Association pour l'avancement des sciences et des techniques de la documentation (ASTED)

\section{ISSN}

0315-2340 (imprimé)

2291-8949 (numérique)

Découvrir la revue

Citer cet article

Marcouiller, F. (1984). Analyse du dépouillement de la revue Documentation et bibliothèques par les index. Documentation et bibliothèques, 30(1), 25-26.

https://doi.org/10.7202/1053598ar

Tous droits réservés (C) Association pour l'avancement des sciences et des techniques de la documentation (ASTED), 1984
Ce document est protégé par la loi sur le droit d'auteur. L'utilisation des services d'Érudit (y compris la reproduction) est assujettie à sa politique d'utilisation que vous pouvez consulter en ligne.

https://apropos.erudit.org/fr/usagers/politique-dutilisation/ 
A la demande du comité de rédaction de la revue Documentation et bibliothèques, nous avons, d'une part, examiné le dépouillement de ce périodique par cinq répertoires signalétiques ${ }^{2}$ en notant le délai et l'exhaustivité du signalement et, d'autre part, vérifié la mention des auteurs de la revue dans le Social Science Citation Index ${ }^{3}$.

\section{Cueillette des données}

Pour chacun des articles et des chroniques (208 au total) parus entre mars 1973 (date de parution du premier numéro de la revue) et juillet 1982 (dernier numéro au moment de cette étude), nous avons colligé sur une fiche les renseignements suivants: nom de l'auteur, titre de l'article, date de parution, signalement par l'un ou l'autre des répertoires concernés et délai du signalement.

La vérification était d'abord faite par nom d'auteur puis par titre. Dans le cas d'une démarche infructueuse, nous procédions alors par sujets (unitermes, mots-clés ou descripteurs, selon les répertoires).

\section{Calcul du délai et de l'exhaustivité}

Nous nous sommes inspirées des travaux de Martyn et Slater ${ }^{4}$ et d'Edwards ${ }^{5}$ portant sur le signalement, par les services secondaires, des publications en bibliothéconomie pour établir le délai et le degré d'exhaustivité.

Le délai représente la différence, exprimée en mois, entre la date de publication du document et la date de parution du répertoire. L'exhaustivité indique en pourcentage le rapport entre le nombre d'articles ou de chroniques signalés par un répertoire donné et le nombre total de ceux parus dans le périodique ${ }^{6}$.

Pour chacune de ces variables, nous avons calculé une moyenne, un écart-type ${ }^{7}$ ainsi que leur corrélation.

\section{Résultats}

Tous les répertoires, sauf $L L$, obtiennent un délai raisonnable, soit de trois à six mois selon Whatley ${ }^{8}$. Pour le répertoire français et les deux répertoires américains, nous pouvons comparer nos résultats à ceux obtenus par Robinson et Turtle dans leur étude de $1974^{\circ}$ qui accordait un

Tableau 1. Délai et exhaustivité par répertoire

\begin{tabular}{lccccc}
\hline & $\begin{array}{c}\text { BS } \\
\text { (France) }\end{array}$ & $\begin{array}{c}\text { CPI } \\
\text { (Canada) }\end{array}$ & $\begin{array}{c}\text { RADAR } \\
\text { (Canada) }\end{array}$ & $\begin{array}{c}\text { LISA } \\
(\mathrm{E}-\mathrm{U})\end{array}$ & $\begin{array}{c}\mathrm{LL} \\
(\mathrm{E}-\mathrm{U})\end{array}$ \\
\hline $\begin{array}{l}\text { Délai } \\
\text { moyen } \\
\text { (en mois) }\end{array}$ & 6,4 & 7 & 8,6 & 7,8 & 26,5 \\
\hline $\begin{array}{l}\text { Écart- } \\
\text { type }\end{array}$ & 2,1 & 3,4 & 4,2 & 4,2 & 6,6 \\
\hline $\begin{array}{l}\text { Exhausti- } \\
\text { vité } \\
\text { moyenne } \\
\text { (en \%) }\end{array}$ & 73,7 & 96,5 & 98,4 & 64,9 & 80,3 \\
\hline
\end{tabular}

1. Cette étude a été réalisée avec la collaboration de Mlle Yélena Valdman dans le cadre du cours BLT-6313 (séminaire de recherche), cours dispensé par Mme Suzanne Gastaldy à l'École de bibliothéconomie de I'Université de Montréal.

2. II s'agit de Bulletin signalétique, section 101: Science de l'information, documentation: Canadian Periodical Index. Radar: répertoire analytique d'articles de revues du Québec; Library and Information Science Abstracts; Library Literature. Par la suite indiqués respectivement par $B S$; $C P I ; R A D A R$; LISA et LL.

3. Par la suite SSCl.

4. J. Martyn et M. Slater, "Tests on Abstracts Journals", Journal of Documentation, vol. 20, no. 4 (1964), 212.

5. T. Edwards, «A Comparative Analysis of the Major Abstracting and Indexing Services for Library and Infor mation Sciences», Unesco Bulletin for Libraries, vol. 30 , no. 1 (1976), 18.
6. Comme nous l'avons indiqué plus haut, nous avions un nombre total de 208 articles et chroniques. De ce nombre, il faut soustraire 10 articles puisque ceux qui sont parus de juillet 1982 à décembre 1982 n'étaient pas encore répertoriés. Ceux parus entre avril et juin 1982 l'étaient uniquement par BS et CPI. Ceux parus en mars 1982 étaient répertoriés dans $B S, C P I$ et $R A D A R$. Cette situation est imputable au délai de signalement de chacun. Nous avons obtenu comme nombre total d'articles et de chroniques pour chaque répertoire: $B S$ (198) CPI(198); RADAR (192); LISA (188); LL (193).

7. Ce calcul a été établi par année et par répertoire et pour la période totale (près de 10 ans).

8. $H$. Whatley, «Les services d'indexation et de résumés analytiques dans le domaine de la bibliothéconomie et de la documentation des bibliothèques", Bulletin de l'Unesco à l'intention des bibliotheques, vol. 19, no 5 (septembre/octobre 1965), 242.

9. M. Robinson et $M$. Turtle, «The relationship between time lag and place of publication in LISA and Library Literature R.Q., vol. 14 (Fall 1974), 28. 
délai de signalement de 3,8 mois pour $L I S A$, 5 mois pour $B S$ (soit trente-cinq jours de plus que $L(S A)$, et de 6,7 mois pour $L L$. Sauf dans ce dernier cas, nos résultats sont donc comparables à ceux obtenus par Robinson et Turtle, si l'on tient compte des écarts-types. La différence, pour les deux premiers répertoires, LISA et $B S$ peut être attribuée à l'échantillonnage.

Quant aux index canadiens $C P I$ et $R A D A R$, il était à prévoir qu'ils obtiendraient des délais de signalement sinon inférieurs du moins comparables aux autres répertoires. Tous deux obtiennent un délai de signalement plus long que $B S$, et dans le cas de RADAR, plus élevé également que LISA. En effet, le délai moyen de signalement est de 7 mois pour $C P I$ et de 8,6 mois pour RADAR.

Au plan de l'exhaustivité, notre étude indique que $98,4 \%$ des articles publiés dans Documentation et bibliothèques ont été signalés par au moins un répertoire. Certaines omissions méritent toutefois d'être notées. Ainsi, pour des raisons que nous ignorons, LISA n'a signalé aucun des articles de la revue parus en 1976. C'est également le cas de $B S$ pour deux numéros en 1974 et 1975 et de $L L$ pour un numéro de 1976. Ces omissions affectent de façon importante le pourcentage d'exhaustivité d'un répertoire.

Malgré cela, en regard de l'étude d'Edwards qui portait spécifiquement sur l'exhaustivité de signalement de Library Resources and Technical Services, Documentation et bibliothèques a obtenu un meilleur score que cette revue. En effet, alors qu'Edwards mentionnait des moyennes d'exhaustivité de $31 \%$ pour $B S, 56 \%$ pour $L / S A$ et $72 \%$ pour $L L$, Documentation et bibliothèques obtient pour sa part des moyennes d'exhaustivité de $73,7 \%$ pour $B S, 64,9 \%$ pour $L I S A$ et $80,3 \%$ pour $L L$.

Quant à $C P I$ et $R A D A R$, dont le mandat est le dépouillement systématique des périodiques, nous pouvions entrevoir un résultat s'approchant de $100 \%$, ce qui fut le cas.

Finalement, le calcul de la corrélation entre nos deux variables (délai et exhaustivité) n'indique rien de significatif.

\section{Social Sciences Citation Index}

La recherche des auteurs de Documentation et bibliothèques dans $S S C /{ }^{10}$ fut réalisée à l'aide d'une liste alphabétique des noms d'auteurs auxquels étaient accolées les dates de parution de leurs articles.

Nous avons ainsi examiné le numéro de $S S C I$ correspondant à l'année de parution de chacun des articles et les trois années subséquentes. Pour compléter cette démarche, nous avons consulté le Source Index du SSC/qui indique les titres de périodiques cités et citants, et le Permuterm Subject Index.

Il résulte qu'aucun des auteurs de Documentation et bibliothèques pas plus que la revue ellemême ne figurent dans cet index.

\section{Conclusion}

Pour les cinq répertoires analysés, le délai (sauf pour $L L$ ) et l'exhaustivité du signalement des articles de Documentation et bibliothèques nous apparaissent satisfaisants et correspondent du moins à la norme habituelle des services secondaires.

L'absence de signalement dans SSC/s'explique sans doute par la moindre importance qu'accordent les chercheurs à Documentation et bibliothèques par rapport à d'autres publications dans le domaine, notamment celles de niveau international. On peut également penser que la revue occupe une position mitoyenne entre la publication scientifique et la publication professionnelle. Les chercheurs ont peut-être tendance à la classer dans cette seconde catégorie.

II conviendrait de considérer certaines solutions permettant d'améliorer le rayonnement de la revue et de ses auteurs à l'extérieur du pays. Sur le plan du contenu, tout en maintenant des articles descriptifs, il serait souhaitable de les axer vers des situations de pratiques nouvelles et originales et d'inclure régulièrement des articles de recherche relativement fouillés. Enfin, de nouveaux efforts de publicité et de diffusion à l'étranger seraient peut-être à envisager.

\section{Francine Marcouiller}

Projet Classification-bibliothèque

Revenu Canada, Impôt

Montréal 